\title{
The emerging role of epigenetics in pulmonary hypertension
}

\author{
Elisabetta Gamen ${ }^{1}$, Werner Seeger ${ }^{1,2}$ and Soni Savai Pullamsetti, ${ }^{1,2}$
}

\begin{abstract}
Affiliations:
${ }^{1}$ Max-Planck-Institute for Heart and Lung Research, Dept of Lung Development and Remodelling, member of the German Center for Lung Research (DZL), Bad Nauheim, Germany.

${ }^{2}$ University of Giessen Lung Center (UGMLC), member of the German Center for Lung Research (DZL),
\end{abstract} Justus-Liebig University, Giessen, Germany.

\section{Correspondence:}

Soni Savai Pullamsetti, Max-Planck-Institute for Heart and Lung Research, Dept of Lung Development and Remodelling, Parkstrasse 1, 61231 Bad Nauheim, Germany.

E-mail: soni.pullamsettiampi-bn.mpg.de

ABSTRACT Epigenetics is usually defined as the study of changes in phenotype and gene expression not related to sequence alterations, but rather the chemical modifications of DNA and of its associated chromatin proteins. These modifications can be acquired de novo, being inherited, and represent the way in which genome and environment interact. Recent evidence points to the involvement of epigenetic changes in the pathogenesis of pulmonary hypertension, as they can partly explain how environmental and lifestyle factors can impose susceptibility to pulmonary hypertension and can explain the phenotypic alteration and maintenance of the disease state.

In this article, we review the epigenetic regulatory mechanisms that are mediated by DNA methylation, the post-translational modifications of histone tails and noncoding RNAs in the pathogenesis of pulmonary hypertension. Furthermore, pharmacological interventions aimed at epigenetic regulators/ modifiers and their outcomes in different cellular and preclinical rodent models are discussed. Lastly, the remaining challenges and future directions in which to explore epigenetic-based therapies in pulmonary hypertension are discussed.

@ERSpublications

Epigenetic mechanisms and pharmacological interventions in pulmonary hypertension http://ow.ly/ykeJ3017fN8

Received: Jan 172016 | Accepted after revision: May 262016 | First published online: Aug 042016

Support statement: This study was funded by the German Research Foundation through Excellence Cluster 147 Cardio-Pulmonary System (ECCPS), Max-Planck-Gesellschaft and the Scientific and Economic Excellence in Hesse (LOEWE) programme. Funding information for this article has been deposited with the Open Funder Registry.

Conflict of interest: None declared.

Copyright @ERS 2016 


\section{Introduction}

Pulmonary hypertension is a progressive disease of multifactorial aetiology, which has a poor prognosis and results in right heart failure. Based on pathogenetic background and histopathological appearance, five categories of pulmonary hypertension have been defined in the latest World Health Organization classification: 1) pulmonary arterial hypertension $(\mathrm{PAH})$; 2) pulmonary hypertension due to left heart disease; 3) pulmonary hypertension due to lung diseases and/or hypoxia; 4) chronic thromboembolic pulmonary hypertension (CTEPH); and 5) pulmonary hypertension with unclear multifactorial mechanisms. Each of these categories encompasses several subcategories [1]. For example, category 1 pulmonary hypertension (PAH) includes idiopathic (IPAH), heritable and drug- and toxin-induced PAH as well as PAH associated with a variety of conditions (including connective tissue diseases, HIV infection, portal hypertension and congenital heart disease), schistosomiasis, pulmonary veno-occlusive disease (PVOD), pulmonary capillary haemangiomatosis and persistent pulmonary hypertension of the newborn (PPHN). When considered together, all variants of pulmonary hypertension may affect up to 100 million people worldwide.

\section{Pathobiology of pulmonary hypertension}

The vascular pathology of pulmonary hypertension is characterised by pulmonary vasoconstriction and by abnormal ("pseudomalignant") inward remodelling processes, which may affect all vessel layers (intima, media and adventitia) [2]. These remodelling processes result in severe loss of the cross-sectional area and a concomitant increase in right ventricular afterload. Intimal changes include initial endothelial injury/ apoptosis, endothelial cell proliferation, invasion of the intima by (myo)-fibroblast-like cells, enhanced matrix deposition, and, in varying degrees, obstruction of the vascular lumen by unique plexiform lesions. Vascular smooth muscle cell (SMC) proliferation is a prominent feature in virtually all pulmonary hypertension entities, causing intimal thickening of the intra-acinar muscular resistance vessels [3, 4] and muscularisation of the normally nonmuscularised precapillary arterioles. In several forms of pulmonary hypertension, these structural abnormalities are accompanied by marked hypertrophy of the adventitia, with (myo)-fibroblast proliferation/invasion and enhanced matrix deposition being observed. Overall, these structural changes suggest a switch from "quiescent" toward "pro-proliferative", "apoptosis-resistant" and "pro-inflammatory" vascular cell phenotypes. As a functional consequence, the pulmonary vascular resistance drastically increases, causing enhanced afterload for the right ventricle with right heart hypertrophy and failure as further cardiac sequelae of the disease [5-7].

\section{Current therapies}

There are three currently approved therapeutic strategies for the treatment of PAH with vasodilatory properties: prostacyclin (prostaglandin $\mathrm{I}_{2}$ ) and its analogues (iloprost, treprostinil and selexipag) [8-11], phosphodiesterase 5 inhibitors (sildenafil and tadalafil) [12, 13] and endothelin receptor antagonists (ERAs) (bosentan) [14]. Further advances have been made with the introduction of novel compounds, such as macitentan and riociguat. The first of these is an orally active ERA with improved tolerability and reduced liver toxicity compared to bosentan $[15,16]$. Riociguat is a soluble guanylate cyclase stimulator, improving nitric oxide production, recently approved by the US Food and Drug Administration to treat adults with $\mathrm{PAH}$ and persistent, recurrent or inoperable CTEPH [17, 18]. Although symptomatic improvement among patients with $\mathrm{PAH}$ is often observed with therapy, haemodynamic changes are typically modest, and most patients continue to have significant pulmonary hypertension despite maximal therapy [19]. Moreover, the unmet clinical need is even more pronounced in pulmonary vascular disorders outside the PAH group, which are more frequent by several orders of magnitude. These include those with underlying heart or lung disease and CTEPH, for which no single medical treatment is currently approved. Hence, deciphering the molecular mechanisms that drive and sustain the maladaptive inward vascular remodelling processes as well as right ventricular maladaptation in pulmonary hypertension is indispensable for the development of therapeutic approaches to prevent or reverse such processes.

Importantly, recent compelling evidence suggests that the vascular cells cultured from hypertensive lung vessels manifest and maintain a hyperproliferative, apoptosis-resistant, pro-inflammatory and pro-fibrotic phenotype when transferred outside the vascular microenvironment, demonstrating the presence of "imprinted" phenotypic changes [20]. It was postulated that these changes could be a consequence of both genetic changes and/or "maladaptive" epigenetic mechanisms (establishment of an "epigenetic memory"), akin to cancer: maladaptive epigenetic mechanisms may include histone modifications (methylation and acetylation) and changes in DNA methylation and noncoding RNA profiles (i.e. changes to the miRome). In this article, we review the epigenetic regulatory mechanisms in the physiology of pulmonary vasculature as well as in the pathogenesis of pulmonary hypertension. Furthermore, the pharmacological interventions aimed at epigenetic regulators/modifiers and their outcomes in different cellular, preclinical rodent models and future directions are discussed. 
Moreover, we focus on the advances made in understanding the roles of epigenetic regulators in the pathogenesis of pulmonary hypertension and the progress toward designing effective treatments targeting the epigenome.

\section{Epigenetics}

Epigenetics is usually defined as the study of changes in phenotype and gene expression not related to alterations in the sequence, but rather the chemical modifications of DNA and of its associated chromatin proteins. These modifications can be acquired de novo, being inherited, and represent the way in which genome and environment interact. Epigenetic modifications are mediated by DNA methylation, post-translational modifications of histone tails and noncoding RNAs, with the first two representing the predominant phenomena. The involvement of these mechanisms in the pathogenesis of many diseases, including cancer and neurodevelopmental and hereditary disorders is well recognised, while, to date, only little evidence of their contribution to pulmonary hypertension is available [21-23].

\section{DNA methylation}

The methylation of DNA is a common mechanism used by cells to "switch off" gene expression. In mammalian cells, DNA methylation occurs at cytosine residues, mainly of CpG dinucleotides, where a methyl group is covalently attached to the C5 position of the nucleobase. This modification is set and maintained by the DNA methyltransferases (DNMT) 1, 3a and 3b. Three principal mechanisms have been shown to be involved in the regulation of gene repression by DNA methylation: 1) the methyl group may directly prevent the binding of transcriptional regulators; 2) it may lead to the specific binding of methyl-CpG binding proteins that interfere with the recruitment of transcription factors; or 3) these methyl-CpG binding proteins may able to recruit large protein complexes, which control the accessibility of DNA through modification of the chromatin structure [24]. CpG sites are distributed unevenly throughout the genome, including all types of sequences: promoter regions, gene bodies, intergenic sequences and repetitive elements. In healthy somatic cells, up to $90 \%$ of $\mathrm{CpG}$ dinucleotides (that is, 3-6\% of all cytosines) are methylated, except for promoter CpG islands, which appear to be protected from methylation. DNA methylation is an important event in numerous physiological cellular processes, including embryonic development, genomic imprinting, $\mathrm{X}$-chromosome inactivation and preservation of chromosome stability, but a growing number of human diseases have been associated with aberrant DNA methylation as well $[25,26]$.

DNA methylation in pulmonary hypertension

Recent literature suggests an involvement of aberrant DNA methylation in the pathogenesis of pulmonary hypertension (figure 1). An increased expression of DNMT1 and DNMT3B and the hypermethylation of the $\mathrm{CpG}$ island in the promoter region of the superoxide dismutase (SOD)2 gene were observed in the lungs and isolated pulmonary arterial SMCs (PASMCs) of fawn-hooded rats, a strain in which PAH occurs spontaneously. This epigenetic silencing of SOD2 contributes to the activation of hypoxia inducible factor (HIF)1 and creates a pro-proliferative, apoptosis-resistant state [27]. Yang et al. [28] demonstrated that reduced levels of global DNA methylation are associated with the loss of the cyclin-dependent kinase inhibitor p21 in the pulmonary vasculature of fetal lambs exposed to high-altitude long-term hypoxia. This may be the cause of the excessive PASMC proliferation observed in these fetuses, leading to pulmonary arterial remodelling and pulmonary hypertension in the newborn lambs. Furthermore, an analysis of genomic DNA extracted from human peripheral blood mononuclear cells and explanted lungs showed increased methylation of the granulysin (GNLY) gene in patients affected by PVOD in contrast to $\mathrm{PAH}$, allowing discrimination between the two groups [29].

\section{Histone modifications}

The eukaryotic genome is organised into a DNA-protein complex known as chromatin, whose basic unit is the nucleosome, an octamer composed by two copies of each of the core histone proteins $(\mathrm{H} 2 \mathrm{~A}, \mathrm{H} 2 \mathrm{~B}, \mathrm{H} 3$ and H4) around which DNA is wrapped. Histones can be post-translationally modified within the 10-30 amino acids at the $\mathrm{N}$-terminal domain (also called the histone "tail") extending from the nucleosomal surface, thus modulating chromatin folding and the binding of regulatory proteins. From a chemical point of view, histone modifications can be divided in two main groups. The first comprises those modifications leading to the addition or removal of small organic substituents (acetylation, methylation, phosphorylation, deamination and palmitoylation), while the second group includes elaborate and large organic molecules (ubiquitylation, SUMOylation, biotinylation, glysosylation and ADP-ribosylation) [30, 31].

\section{Histone acetylation}

Among these, acetylation was the first described and is one of the most widespread modifications of histones. It involves the addition of an acetyl group, catalysed by histone acetyltransferases (HATs) from the cofactor acetyl-CoA to a lysine residue of the histone tail, especially those in H3 and H4. Generally, 

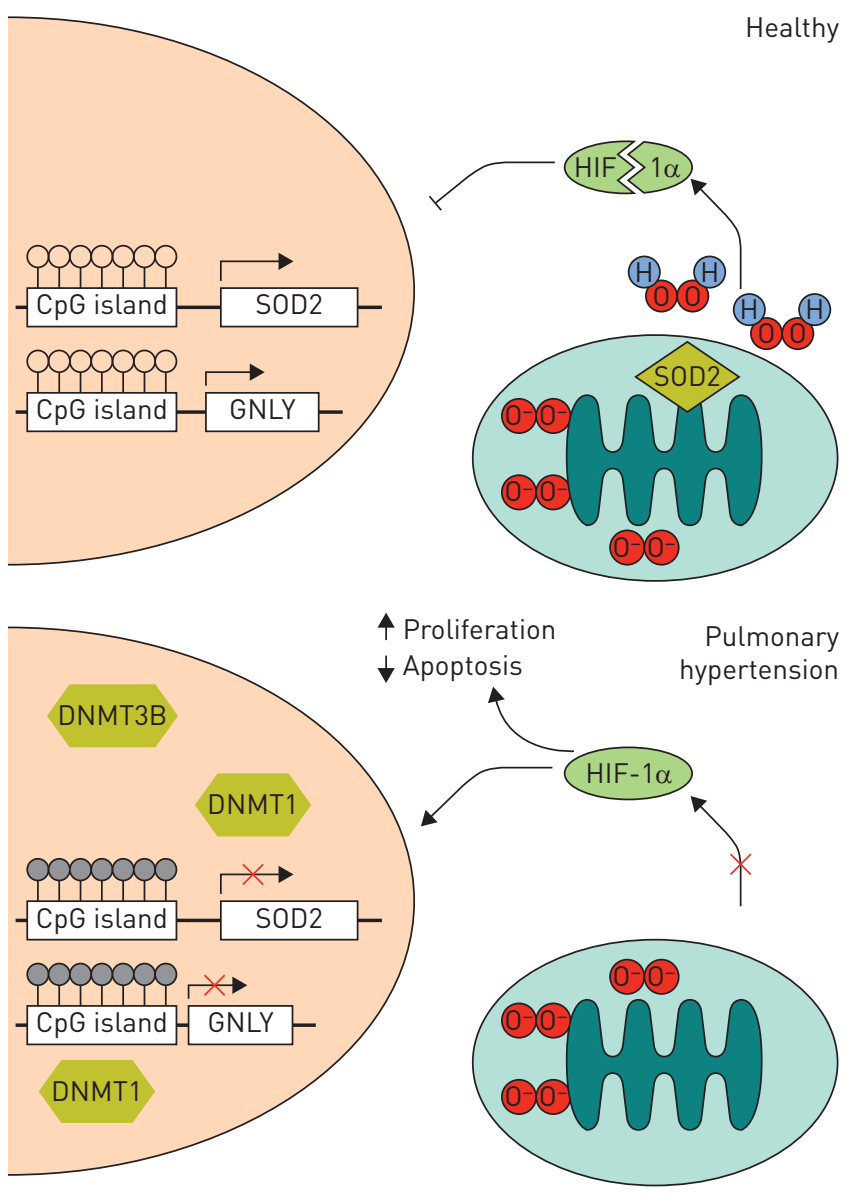

FIGURE 1 Mechanism for epigenetic regulation of mitochondrial superoxide dismutase (SOD)2 in pulmonary arterial smooth muscle cells: the higher expression of DNA methyltransferase (DNMT)1 and DNMT3B leads to the hypermethylation of $\mathrm{CpG}$ islands in the promoter of the SOD2 gene and its downregulation. This impairs $\mathrm{H}_{2} \mathrm{O}_{2}$-mediated redox signalling, activates hypoxia-inducible factor (HIF)-1 $\alpha$, and creates a proliferative, apoptosis-resistant state. DNA hypermethylation is also responsible for the epigenetic silencing of the granulysin (GNLY) gene.

this modification neutralises the positive charge of lysine residues, weakening the charge-dependent interactions between histone and DNA and increasing the accessibility to the transcription machinery [32]. Conversely, histone deacetylation favours transcriptional repression by allowing chromatin compaction. Acetylated histones can also act as binding sites for the transcriptional co-activator proteins that may further modify the chromatin structure [33]. Apart from histone acetylation, transcription factors can be directly acetylated and deacetylated, which can have positive and negative consequences on gene transcription [34]. Importantly, acetylation is not a permanent modification, but instead a dynamic process determined by the antagonistic actions of two large families of enzymes: HATs and histone deacetylases (HDACs) (table 1) [32].

\section{Histone acetylation in pulmonary hypertension}

Aberrant histone acetylation and deacetylation have been described in pulmonary hypertension. For example, an increased $\mathrm{H} 3$ and $\mathrm{H} 4$ acetylation at the proximal promoter region of endothelial nitric oxide synthase (eNOS), which leads to increased levels of eNOS, is shown in pulmonary arterial endothelial cells (PAECs) isolated from PPHN rat model [35]. This study suggests that the epigenetic regulation of eNOS plays an important role in the pathogenesis of PPHN. In fibroblasts isolated from the distal pulmonary arteries of chronically hypoxic hypertensive calves an increased expression and activity of class I HDACs (HDAC1, HDAC2 and HDAC3) was observed, and the specific inhibition of these deacetylases was able to suppress the production of pro-inflammatory mediators [36]. Similar findings were observed in the PASMCs of fetal lambs exposed to high-altitude long-term hypoxia. In fact, the level of H4 acetylation, together with global DNA methylation, was reduced, and treatment with the HDAC inhibitor trichostatin A (TSA) was able, on one hand, to suppress platelet-derived growth factor (PDGF)-induced PASMC 
TABLE 1 Classification of histone acetyltransferases (HATs) and histone deacetylases (HDACs)

\begin{tabular}{|c|c|c|}
\hline Category & Name & Involvement in diseases \\
\hline \multirow{3}{*}{ HAT type A } & MYST & Lung and kidney cancer, cardiac hypertrophy and ataxia telangiectasia \\
\hline & Basal TF & Cancer \\
\hline & NRCF & Cancer \\
\hline \multirow{2}{*}{ HAT Type B } & HAT 4 & N/A \\
\hline & (Naa60) & \\
\hline \multirow[t]{3}{*}{ HDACs class I } & HDAC1 & $\begin{array}{c}\text { Cancer (breast, prostate and ovarian), hepatocellular carcinoma, hepatitis, leukaemia and } \\
\text { Huntington's disease }\end{array}$ \\
\hline & HDAC2 & Cancer (prostate, pancreas, lung and colon) and hepatocellular carcinoma \\
\hline & HDAC3 & Cancer (breast, lung and colon), leukaemia, multiple sclerosis and Huntington's disease \\
\hline \multirow[t]{2}{*}{ HDACs class IIb } & HDAC6 & Cancer, leukaemia and multiple and amyotrophic lateral sclerosis \\
\hline & HDAC10 & Lung cancer and schizophrenia \\
\hline \multirow[t]{7}{*}{ HDACs class III (sirtuins) } & SIRT 1 & $\begin{array}{c}\text { Cancer, leukaemia, multiple and amyotrophic lateral sclerosis, bronchopulmonary dysplasia and } \\
\text { Parkinson's disease }\end{array}$ \\
\hline & SIRT 2 & Cancer, Parkinson's and Huntington's diseases, obesity and diabetes \\
\hline & SIRT 3 & Cancer, Alzheimer's disease, obesity and diabetes mellitus \\
\hline & SIRT 4 & B-cell lymphoma and diabetes mellitus \\
\hline & SIRT 5 & Cancer (breast, pancreatic and head and neck) and schizophrenia \\
\hline & SIRT 6 & Cancer (colon, breast and pancreatic) \\
\hline & SIRT 7 & Breast and thyroid cancer and hepatocellular carcinoma \\
\hline HDACs class IV & HDAC11 & Breast cancer, Hodgkin's lymphoma, amyotrophic lateral sclerosis and Huntington's disease \\
\hline
\end{tabular}

HATs are categorised into two types: type A (nuclear) and type B (cytoplasmic). Type A HATs are further divided into five families: GNAT, p300/ CBP, MYST, basal transcription factors (TF) and nuclear receptor cofactors (NRCF). Type B HATs are further divided into HAT1, HAT2 and HAT4. HDACs are divided into four major subclasses: I, Ila, Ilb and IV (zinc-dependent) and class III, also called sirtuins (SIRT; nicotinamide adenine dinucleotide-dependent).

proliferation and migration and, on the other, to restore the DNA methylation, suggesting an interplay between histone modifiers and DNA methylation [28]. Recently, the mitochondria-localised deacetylase sirtuin (SIRT)3 expression was found to be reduced in human IPAH PASMCs [37]. Using SIRT3 knockout mice, the authors showed that the decreased SIRT3 expression is associated with the suppression of mitochondrial function, inhibition of apoptosis and activation of several pulmonary hypertension-related transcription factors, such as HIF1 $\alpha$, signal transducer and activator of transcription (STAT) 3 and nuclear factor of activated T-cells (NFAT)c2 [37]. In addition, transgenic mice lacking SIRT1 catalytic activity exhibited greater susceptibility to pulmonary hypertension in response to chronic hypoxia (personal communication; M. Taha, University of Ottawa, Ottawa, Canada). PAH-PASMCs were shown to have an activation of poly(ADP-ribose) polymerase (PARP)1, a critical enzyme implicated in DNA repair, allowing proliferation despite the presence of DNA-damaging insults, eventually leading to PAH [38]. As SIRT1 and PARP1 are competing for nicotinamide adenine dinucleotide, increased PARP1 can explain the decreased SIRT1 activity that may subsequently promote PAH.

In line with these findings, several compounds with HDAC inhibitory activity have been explored as therapeutic strategies for pulmonary hypertension. Valproic acid (VPA), a class I HDAC inhibitor used as an antiepileptic and anticancer drug, and suberoylanilide hydroxamic acid (SAHA), a broad-spectrum HDAC inhibitor approved for the treatment of T-cell lymphoma, were shown to be therapeutically beneficial in experimental models of pulmonary hypertension. In fact, treatment with VPA or SAHA, even after the establishment of pulmonary hypertension, reduced mean pulmonary arterial pressure, right ventricular hypertrophy and vascular remodelling [39]. More recently, sodium butyrate, a pan-HDAC inhibitor with antineoplastic/antiproliferative properties was also shown to be able to reduce in vitro PDGF-induced human PASMC proliferation and migration [40]. In contrast, treatment with TSA, a 
broad-spectrum HDAC inhibitor, did not decrease the already established right ventricle hypertrophy in a rat pulmonary artery banding $(\mathrm{PAB})$ model, but instead further reduced the cardiac output and increased dilatation, leading to right ventricle failure [41], implying that HDAC inhibitors can give rise to unwanted side-effects, which can be partly explained by the additional pharmacological activities of these inhibitors. For example, VPA was shown to regulate ion channels, glycogen synthase kinase- $3 \beta$ and mitogen-activated protein kinases [42]. For these reasons, selective small-molecule inhibitors of HDACs have also been investigated. MGCD0103, highly selective for class I HDACs (HDAC1, HDAC2 and HDAC3), and MS-275, highly selective for HDAC1, were used in a chronic hypoxia-induced pulmonary hypertension rat model in which they were able to reduce pulmonary arterial pressure and only modestly reduce right ventricular hypertrophy [43]. In contrast, treatment with resveratrol, a SIRT1 (a class III HDAC) pathway activator, was shown to both prevent and attenuate monocrotaline-induced pulmonary hypertension in rats due to its antiproliferative and anti-inflammatory effects on PASMCs [44, 45]. At the current stage of knowledge, it seems clear that in vivo studies involving the modulation of HDACs by more or less specific inhibitor compounds lead to unpredictable "off-target" effects, accentuating the need for nonpharmacological validation approaches, such as the use of genetically modified animals.

Histone methylation

Histone methylation involves the addition of methyl groups to lysine or the arginine residues of histone tails using $S$-adenosyl methionine as a methylating agent. It has a high degree of selectivity and possesses a dual role in gene expression, activating or repressing gene expression, depending on the methylated residue and the number of attached methyl groups. Lysine can be mono-, di- or trimethylated by histone methyltransferase (HMT or PKMT), while arginine residues can only be mono- or dimethylated by protein arginine methyltransferases. Like acetylation, histone methylation is a dynamic process, and the removal of the methyl groups is mediated by demethylases. Two evolutionarily conserved families of histone demethylases (the LSD demethylases and the JMJC demethylases), which utilise different reaction mechanisms to establish demethylation, have been identified [31].

\section{Histone methylation in pulmonary hypertension}

Histone methylation is less explored than histone acetylation in pulmonary hypertension. Increased expression of the enhancer of zeste homologue (EZH)2, which has an HMT activity, was found in proliferating PASMCs and associated with progression of pulmonary hypertension in a hypoxia-induced mouse model [46]. Similarly, the treatment of ovine fetal PASMCs with BIX-01294, a specific inhibitor of the HMT G9a (an enzyme responsible for H3K9me2/histone H3 dimethylation at position lysine-9) was shown to reduce PDGF-induced proliferation, migration and contractility in the above-mentioned cells [47].

\section{Noncoding RNAs}

Noncoding RNAs are transcripts of genes not encoding for proteins. They are divided into two classes based on an arbitrary length cut-off. Those $<200$ nucleotides in length are referred to as small noncoding RNAs, while those with more than this are known as long noncoding RNAs [48].

\section{MicroRNAs}

MicroRNAs (miRNAs) are small noncoding RNA molecules of $\sim 22$ nucleotides, regulating the post-translational modifications of gene expression, thus playing an important role in development and in physiological processes [49]. During miRNA biogenesis, a long primary transcript (pri-miRNA) is generated in the nucleus and subsequently converted by the RNAse III endonuclease Drosha into a smaller molecule of 60-70 nucleotides (pre-miRNA). It is then transported into the cytoplasm, where it is again processed by another RNA endonuclease, named Dicer, giving rise to the mature miRNA duplex. This molecule is rapidly incorporated into a ribonucleoprotein complex, known as the RNA-induced silencing complex, containing members of the conserved Ago protein family, and unwound. Only one of the strands (guide strand) is stably associated with the Ago protein, whereas the other one (passenger strand) is discarded [50]. This miRNA recognises and binds the so called "seed" sequence located in the 3' untranslated terminal regions (UTRs) of the mRNAs. The degree of this complementarity plays a fundamental role in the regulatory mechanism: a perfect match allows the Ago-catalysed cleavage of the mRNA strand, whereas a central mismatch promotes the repression of mRNA translation [49].

Thousands of miRNAs across different species have been identified, and a nomenclature system has therefore been adopted [51]. miRNAs are named using the "mir" prefix followed by a dash and a unique identifying number that is assigned sequentially. let- 7 and lin- 4 are obvious exceptions, and these names are retained for historical reasons [51]. The latest miRBase release, 21 (R21), contains 28645 experimentally validated miRNA genes expressing 35828 mature miRNA in 223 species [52]. Moreover, miRNA genes have been clustered based on intergenic distance; they are considered to be in a cluster when they are situated in a 
region typically $<1-2 \mathrm{~kb}$ [53]. Several experimental studies suggest a relationship between the expression of aberrant miRNAs and the pathogenesis of a variety of diseases, ranging from diabetes mellitus to cardiac hypertrophy and from cancer to pulmonary hypertension. Lu et al. [54] found evidence that miRNAs derived from clustered miRNA genes tend to have similar functional roles and disease associations.

\section{MicroRNAs in pulmonary hypertension}

The first evidence of the involvement of miRNAs in the pathogenesis of pulmonary hypertension came from a screening of rat lungs after exposure to chronic hypoxia or monocrotaline. A reduced expression of Dicer in both animal models and the downregulation of miR-22, miR-30 and let-7f was identified, whereas miR-322 and miR-451 were found to be upregulated [55]. The same regulation was observed in vitro by using rat pulmonary arterial fibroblasts (PAFs) and PASMCs as well as human PAFs and PAECs exposed to hypoxia and/or the growth factors involved in pulmonary hypertension, such as transforming growth factor- $\beta$ and bone morphogenetic protein (BMP). Furthermore, the miR-21 downregulation observed in the monocrotaline model was confirmed in human lung tissues and serum from patients with IPAH [55]. Nevertheless, several differences were observed between the two animal models, for example, miR-21 and let-7a expression; this is not completely unexpected considering the differential pathobiology induced by hypoxia and monocrotaline [55]. This feature has been addressed in a successive study where the expression pattern of seven miRNAs that were already reported to be involved in the development of pulmonary hypertension was analysed between three different rodent models (monocrotaline- and hypoxia plus SU5416-exposed rats and hypoxia-exposed mice) and human samples [56]. In this study, marked disparities in the expression of the miRNAs were reported, mostly in plasma samples, where only miR-424 showed concordant changes between the human and at least one animal model [56]. Recently, BERTERO et al. [57] showed that the miR-130/131 family is induced in multiple models of pulmonary hypertension as well as in human samples, giving us a first description of a functional hierarchy of miRNAs relevant to pulmonary hypertension, as the family is able to repress miR-204 and miR-424/503. In the context of CTEPH, CHEN et al. [58] proved that the susceptibility to the disease conferred by a deletion/insertion polymorphism in the $3^{\prime}$ UTR of the fibrinogen- $\alpha$ gene (FGA) involves miR-759. In fact, it targets the polymorphic allele and decreases the stability of the FGA mRNA. Microarray analysis of human plasma samples has revealed 35 differentially expressed circulating miRNAs in CTEPH samples. Particularly, let-7b expression is reduced in CTEPH samples, compared to donors, and inversely correlates with the plasma levels of vasoconstrictor endothelin- 1 and transforming growth factor- $\beta$ receptor- 1 expression in PAECs. Through the modulation of these molecules, let-7b regulates the migration of PAECs and PASMCs [59].

Many miRNAs are expressed in a cell- and tissue-specific manner, controlling the proliferation, apoptosis and differentiation of different cell types. In recent years, several studies have explored the regulation and putative role of miRNAs in the physiology and pathophysiology of pulmonary circulation. Although it is a challenge to attribute miRNA effects to specific cell types, for comprehensibility, we ascribed miRNA effects to specific vascular cell types (PAECs, PASMCs and PAFs) (table 2). In fact, to date, the miRNAs previously linked to pulmonary hypertension have been associated primarily with discrete cell-specific mechanisms and phenotypes. miRNAs involved in pulmonary hypertension are summarised in table 3 and figure 2.

MicroRNAs in PAECs

Analysis of miRNAs expressed in endothelial cells has revealed that miR-21 is one of the most highly expressed miRNAs in these cells [83]. Recently, it was shown that in healthy endothelium, miR-21 represses the programmed cell death (PDCD) 4 factor/caspase-3 pathway, controlling endothelial apoptosis. In fact, in miR-21 $1^{-/-}$mice, the constitutive activation of the PDCD4/caspase- 3 axis in PAECs led to the onset of pulmonary hypertension, whereas mice that overexpress miR-21 are partially resistant to chronic hypoxia plus Su5416-induced pulmonary hypertension [84]. Moreover, in human PAECs, interleukin-6

\begin{tabular}{|c|c|c|c|}
\hline & Targets & Animal models & References \\
\hline VPA & Class I & PAB, MCT, Hox rats & {$[39,40]$} \\
\hline SAHA & Class I, II and IV & Hox rats & [39] \\
\hline TSA & All HDACs & PAB & [41] \\
\hline MGCD0103 & Class I & Hox rats & [43] \\
\hline MS-275 & Class I & Hox rats & [43] \\
\hline
\end{tabular}

VPA: valproic acid; SAHA: suberoylanilide hydroxamic acid; TSA: trichostatin A; PAB: pulmonary artery binding; MCT: monocrotaline-induced pulmonary hypertension; Hox rats: chronic hypoxia-induced rat model. 


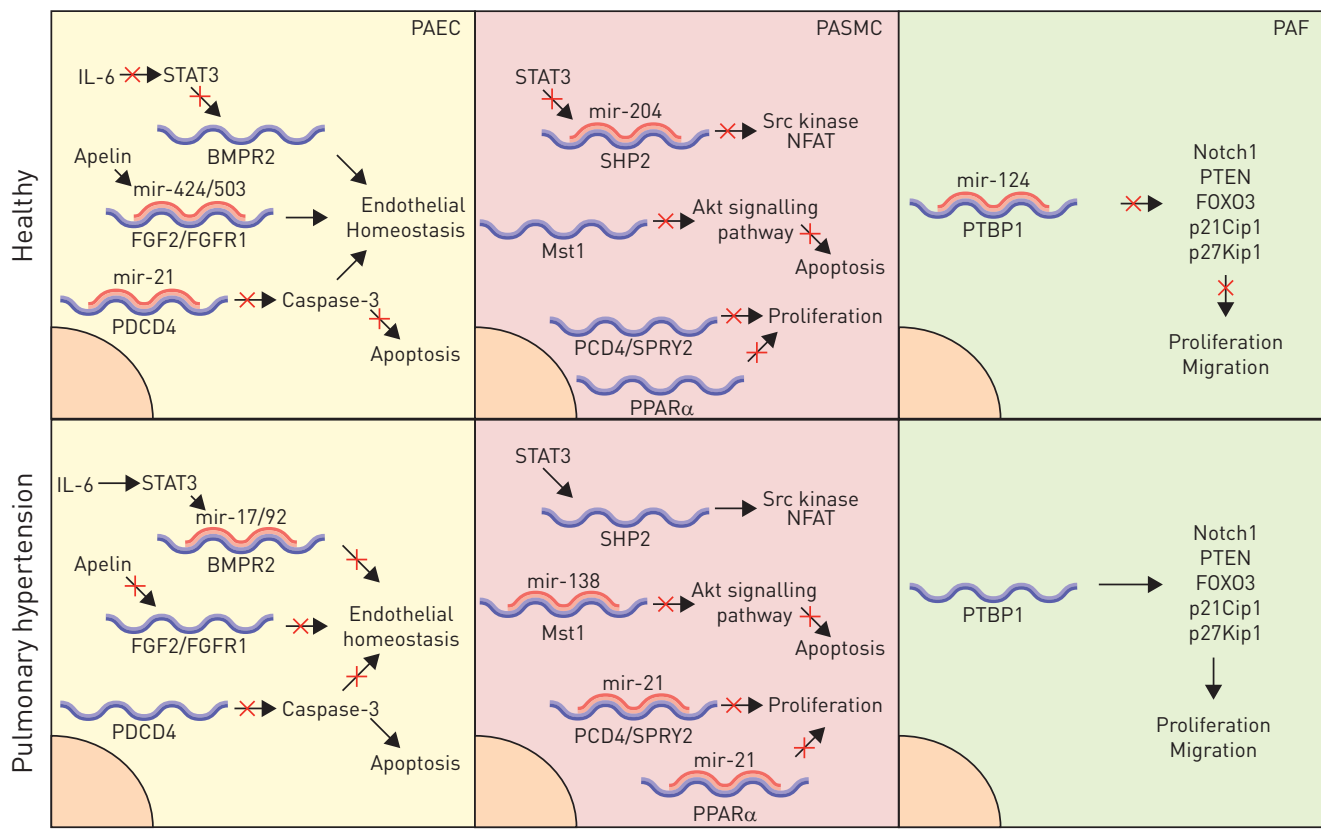

FIGURE 2 MicroRNAs associated with pulmonary hypertension. Many microRNAs are expressed in a cell- and tissue-specific manner, controlling the proliferation, apoptosis and migration of different cell types. PAEC: pulmonary arterial epithelial cell; PASMC: pulmonary arterial smooth muscle cells; PAF: pulmonary arterial fibroblasts; STAT: signal transducer and activator of transcription; BMPR: bone morphogenetic protein receptor; FGF: fibroblast growth factor; FGFR: FGF receptor; PDCD: programmed cell death protein; SHP: Src homology 2 domain-containing phosphatase; NFAT: nuclear factor of activated T-cells; PPAR: peroxisome proliferator-activated receptor; SPRY: sprouty; PTBP: polypyrimidine tract-binding protein; PTEN: phosphatase and tensin homologue.

regulates the production of vasoactive factors, such as endothelin-1, by controlling peroxisome proliferator-activated receptor (PPAR)- $\gamma[67]$.

\section{MicroRNAs in PASMCs}

miRNAs also play an important role in SMCs. In fact, the cell-specific deletion of Dicer is embryonically lethal and associated with reduced SMC proliferation and differentiation, causing reduced contractility and haemorrhages [87]. miR-21 expression was found to be induced by hypoxia in human PASMCs, and, through the post-transcriptional suppression/downregulation of its unique targets such as PDCD4, sprouty (SPRY)2 and PPAR $\alpha$, it regulates cell proliferation and migration [88]. Our group has demonstrated similar pro-proliferative effects and p21 regulation by miR-17 in PASMCs as observed in PAECs [61].

Courboulin et al. [68] demonstrated miR-204 downregulation in chronic hypoxic and monocrotaline-exposed rat lung as well as in human samples, where it correlates with disease severity, thus supporting its possible use as a biomarker for PAH. STAT3 activation suppresses miR-204 expression and the consequent upregulation of its target bromodomain-containing protein (BRD)4 [69]. These changes are in part responsible for the activation of transcription factors such as NFAT and HIF1 in cultured PAH-PASMCs, enhancing their proliferation and resistance to apoptosis [68]. Importantly, the administration of synthetic miR-204 [68] or the pharmacological inhibition of BRD4 [69] significantly reduced disease severity in animal models of pulmonary hypertension. Moreover, the miR-130/301 family was shown to control the STAT3-miR-204 axis by repressing PPAR $\gamma$ [57].

Similarly, miR-206 was found to be significantly downregulated in lung tissues from a hypoxia-induced Sprague Dawley rat model of pulmonary hypertension and in hypoxia-exposed PASMCs. However, there was no correlation between miR-206 downregulation and the duration of hypoxia exposure in vivo, suggesting that it could be involved in the onset stage of hypoxia-induced pulmonary hypertension [70]. miR-206 directly binds the HIF-1 $\alpha \quad 3^{\prime}$-UTR and affects the expression of the Fhl (four and a half LIM domains)-1 protein, a HIF1 $\alpha$ target already known to be elevated in pulmonary hypertension and involved in the regulation of the migration and proliferation of PASMCs [89]. In hypoxic conditions, HIF1 $\alpha$ is responsible for the mir-9 upregulation that is involved in the hypoxia-induced dedifferentiation of PASMCs by repressing multiple endogenous SMC marker genes and by promoting proliferation [72]. miR-138 expression is also increased by hypoxia via HIF1 $\alpha$ and, through the negative regulation of the 
serine/threonine kinase, mammalian sterile 20-like kinase (Mst)1 contributes to the activation of the Akt pathway and the regulation of apoptosis of PASMCs by preventing caspase activation [73]. miR-223 expression is reduced in human lungs from hypertensive patients and specifically in PASMCs due to the normoxic activation of HIF1 $\alpha[75,76]$. miR-223 downregulation promotes poly(ADP-ribose)polymerase (PARP)1 expression, a well-known player in the DNA repair machinery and highly activated in pulmonary hypertension, contributing to cell proliferation and apoptosis resistance [75]. miR-223 also affects vascular remodelling by negatively regulating the insulin-like growth factor-1 receptor [77], RhoB and myosin light chain (MCL)2 expression [76]. In line with these findings, hypoxia-exposed miR-223 knockout mice showed a more pronounced pulmonary hypertension phenotype [77], and its ectopic delivery reverses pulmonary hypertension in the monocrotaline rat model $[75,76]$

In silico analysis performed on PASMCs isolated from CTEPH patients revealed 18 differentially expressed miRNAs. Among them, let-7d showed the strongest downregulation and has been implicated in cell proliferation by regulating $\mathrm{p} 21$ [78].

\section{MicroRNAs in PAFs}

WANG et al. [79] showed that mir-124 expression is reduced in fibroblasts isolated from calves and humans with severe pulmonary hypertension contributing to the highly proliferative, migratory and inflammatory phenotype through the modulation of monocyte chemotactic protein-1 expression. The effects of mir-124 are mediated via direct binding to the mRNA of polypyrimidine tract-binding protein (PTBP) 1 and the subsequent regulation of Notch1/PTEN/FOXO3/p21Cip1 and p27Kip1 signalling. Interestingly, HDAC inhibitors restored miR-124 levels to near normal and reversed the characteristics of the activated phenotype. These findings suggest a mechanistic link between HDACs and miRNAs. Another study claims that mir-124 is also downregulated by hypoxia in human PASMCs, acting as an inhibitor of the NFAT pathway [80].

\section{MicroRNAs in the right ventricle}

Right ventricular failure is the major cause of mortality for patients with pulmonary hypertension. Several groups have been working on understanding the underlying molecular mechanisms and have provided evidence of deregulated miRNAs in the right ventricle.

PAULIN et al. [82] determined the expression of 377 miRNAs in the right ventricle of a monocrotaline rat model and found that the myocardium-specific miR-208 was reduced during the progression of right ventricle hypertrophy. miR-208 downregulation in right ventricle cardiomyocytes is mediated by myocyte enhancer factor (Mef)2c, a transcription factor essential for heart development and, along with tumour necrosis factor- $\alpha$, leads to an increase in the Med13-NcoR1 axis, resulting in Mef2 repression. Thus, Mef2 is not able to promote compensation and triggers the entrance of right ventricle cardiomyocytes into the decompensation phase [82].

In the hypoxia-induced pulmonary hypertension mouse model, SHI et al. [77] reported decreased miR-223 expression in right ventricle tissues, and its cardiac-specific restoration using an adeno-associated virus overexpression of miR-223 improved cardiac function. In addition, in human heart samples, one study showed an association between miR-126 downregulation and right ventricle failure [81]. In right ventricle tissue-derived endothelial cells, miR-126 expression is reduced by DNA hypermethylation, causing the induction of SPRY-related EVH1 domain-containing protein (SPRED)1, a negative regulator of the vascular endothelial growth factor signalling pathway [81]. These studies point out the crosstalk of DNA methylation and miRNA expression in the initiation and progression of pulmonary hypertension and right ventricle dysfunction.

\section{Long noncoding RNAs}

Long noncoding RNAs (lncRNAs) are noncoding RNA molecules (>200 nucleotides) involved in controlling gene expression by functioning as scaffolds for chromatin-modifying complexes and nuclear bodies and as enhancers and mediators of long-range chromatin interactions $[48,90]$. Several lncRNAs have been identified in humans and mice, and their aberrant regulation has been associated with various diseases [91-96]. In the context of pulmonary hypertension, only lately has their role begun to be investigated. Microarray analysis in lung tissues from a hypoxic pulmonary hypertension rat model has revealed a total of 362 differentially expressed lncRNAs [97]. With the same approach, 185 lncRNAs were found to be dysregulated in the pulmonary artery endothelium samples of CTEPH patients as compared with healthy controls [98]. Recently, increased expression of the lncRNA, named NONHSAT07364, was observed in CTEPH lungs and was involved in the angiogenic process, similar to the platelet-activating factor acetyl hydrolase 1B1 (PAFAH1B1), with which it shares 92\% similarity [99]. 


\section{Research challenges and novel ideas/hypotheses}

Pulmonary hypertension is a complex disease that is mediated by the interplay of a predisposed genetic background, an epigenetic state and injurious events. As discussed above, recent evidence points to the involvement of epigenetic changes in the pathogenesis of pulmonary hypertension. These epigenetic changes in part may explain how environmental (hypoxia and altitude) and lifestyle factors (diet, phentermine-fenfluramine and amphetamines) can impose aberrant gene expression patterns in an individual's lifetime that can result in increased risk of pulmonary hypertension. As mentioned, apart from genetic changes, epigenetic changes may result in the phenotypic alteration and maintenance of the disease state. Previous studies have demonstrated that epigenetics is closely associated with the development of hypoxic PAH following intrauterine growth retardation/maternal undernutrition during pregnancy. In addition, brahma-related gene (Brg) 1 and brahma (Brm), two catalytic components of the mammalian chromatin remodelling complex and components of the histone $\mathrm{H} 3$ lysine 4 methyltransferase complex, provide the crucial epigenetic link to the induction of hypoxia-induced cell adhesion molecules and leukocyte adhesion that engenders the endothelial malfunction and pathogenesis of hypoxia-induced pulmonary hypertension [100-102].

As described earlier, extensive analysis of miRNA regulation in pulmonary hypertension has been performed. With regard to histone modifications, we are just starting to comprehend the regulation of histone-modifying enzymes; that is, gene expression in disease states, mostly from human lung tissue and in lungs and right ventricles from rats exposed to hypoxia [39]. Li et al. [36] have shown the increased expression and activity of class I HDACs in fibroblasts from the pulmonary arteries of chronically hypertensive calves. As these changes can happen in a site-specific manner, systemic evaluation of compartment- and cell-specific histone modifying enzymes (HDACs, HAT, HMT and others) in experimental and human pulmonary hypertension and the isoform selective functions/role of these modifying enzymes in pulmonary vascular and right ventricle remodelling need to be conducted in detail. We have to understand how the combination of different histone modifications (acetylation and methylation) appearing on different histones at different times can mediate a unique cellular response, referred as the "histone code", employing novel and high-throughput technologies and methods such as chromatin immunoprecipitation (ChIP) sequencing, ChiP-ChiP and DNA methylation status employing bisulfite sequencing. Improved resources and methodologies would allow epigenome mapping in normal and disease states and will provide key mechanistic insights into perturbed regulatory pathways.

Importantly, a better understanding of the intricate intersecting pathways between DNA methylation, miRNAs and histone modifications will be critical, because, in recent years a growing number of studies, mostly from the cancer field, have demonstrated that epigenetic modifications do not function alone, but instead work together and can cross-regulate each other. Early studies have demonstrated a link between DNA methylation and histone modifications mediated by a group of proteins that specifically recognise methylated CpG sites, called methyl-CpG binding proteins. They are able to bind to methylated DNA and recruit a protein complex containing HDACs and HMTs, leading to a DNA-methylation-induced change of the chromatin structure [103-105]. In particular, genome-scale DNA methylation studies have shown a correlation between DNA methylation and the absence of $\mathrm{H} 3 \mathrm{~K} 4$ methylation and vice versa, probably due to the de novo Dnmt3a specifically recognising unmethylated H3K4 [106, 107]. Similarly, miRNAs can direct DNA methylation at loci from which they are produced as well as in trans at their target genes [108]. They can also play an important role in the control of DNA methylation or histone modification. Understanding these regulatory networks will enhance our knowledge of the epigenetic contribution to disease, thereby enabling the discovery of novel preventative and treatment strategies.

Modifications of DNA and histones are reversible, making them good targets for therapeutic intervention [109]. In corroboration, the inhibition of HDACs, miRNAs and DNMTs have gained attention as potential therapeutic targets. However, for epigenetic modulation therapies, the lack of specificity for treatment is an important issue. For example, the increased global DNA demethylation via the use of nonselective DNMT inhibitors can contribute to increased chromosomal instability with the possibility of being transmitted between generations [109]. Therefore, developing DNA methylation inhibitors that target specific genes or groups of genes associated with pulmonary hypertension is worth further investigation. As miRNA inhibitors are currently being tested for different indications in clinical trials [110] (https://clinicaltrials. gov/ct2/results?term=microRNA\&Search=Search), the clinical development as a new therapy for pulmonary hypertension might therefore be imminent. It will be important to define, for potential therapeutic purposes, the optimal mimic delivery strategy that leads to effective and safe administration to the pulmonary vascular compartment in vivo.

In contrast, the role of HDACs and the use of their inhibitors in the context of pulmonary hypertension are still controversial. Treatment with the HDAC inhibitors VPA and SAHA as well as class I HDAC inhibitors was able to reduce pulmonary arterial pressure, right ventricular hypertrophy and vascular remodelling in 
experimental models of pulmonary hypertension [39, 43]. KIM et al. [111] have shown that myocyte enhancer factor 2-increased activity in PAH PAECs is due to the nuclear accumulation of HDAC4 and HDAC5 and that their pharmacological inhibition is able to rescue experimental pulmonary hypertension models.

However, one major issue raised about the therapeutic potential of HDAC inhibitors in pulmonary hypertension is their unwanted side-effects on the right ventricle. BOGAARD et al. [41] have described reduced HDAC activity in lung tissues combined with increased activity in the hypertrophic hearts of pulmonary hypertension model animals. Furthermore, they showed that the use of the pan-inhibitor TSA has no beneficial effects, but that it worsens right ventricular function and increases cardiac fibrosis in PAB rat models. This can be due to their lack of specificity, in particular their lack of isoform selectivity, and lack of known targets. Thus, the isoform-selective HDAC inhibitors need to be evaluated for preclinical and clinical efficacy. Similarly, we need to resolve HDAC interactions with other nonhistone proteins and identify the genomic targets of HDAC complexes in the disease state so that new therapies can be directed toward HDAC complexes. Moreover, we need to learn about the different ways that HDAC inhibitors affect gene expression; that is, the HDAC-mediated and non-HDAC-mediated effects of pan-HDAC inhibitors need to be studied in detail. Drugs that target HMT and HDMs enzymes are considered more specific than HDACs in that they target fewer residues [112]. However, work on these enzymes is still in its infancy and more work is necessary to determine their specificities and the stabilities of the changes they exert. Furthermore, a deep understanding of the exact mechanisms of the gene regulation involved in vascular and cardiac remodelling in pulmonary hypertension is essential for the development of specific pharmacological modulators that will allow the development of more precise therapeutic strategies.

\section{References}

1 Simonneau G, Gatzoulis MA, Adatia I, et al. Updated clinical classification of pulmonary hypertension. J Am Coll Cardiol 2013; 62: Suppl. 25, D34-D41.

2 Tuder RM, Archer SL, Dorfmüller P, et al. Relevant issues in the pathology and pathobiology of pulmonary hypertension. J Am Coll Cardiol 2013; 62: Suppl. 25, D4-12.

3 Hoffmann J, Marsh LM, Pieper M, et al. Compartment-specific expression of collagens and their processing enzymes in intrapulmonary arteries of IPAH patients. Am J Physiol Lung Cell Mol Physiol 2015; 308: L1002-L1013.

4 Stacher E, Graham BB, Hunt JM, et al. Modern age pathology of pulmonary arterial hypertension. Am J Respir Crit Care Med 2012; 186: 261-272.

5 Rabinovitch M. Molecular pathogenesis of pulmonary arterial hypertension. J Clin Invest 2012; 122: 4306-4313.

6 Seeger W, Pullamsetti SS. Mechanics and mechanisms of pulmonary hypertension - conference summary and translational perspectives. Pulm Circ 2013; 3: 128-136.

7 Pullamsetti SS, Schermuly R, Ghofrani A, et al. Novel and emerging therapies for pulmonary hypertension. Am J Respir Crit Care Med 2014; 189: 394-400.

8 Olschewski H, Simonneau G, Galiè N, et al. Inhaled iloprost for severe pulmonary hypertension. $N$ Engl J Med 2002; 347: 322-329.

9 McLaughlin VV, Benza RL, Rubin LJ, et al. Addition of inhaled treprostinil to oral therapy for pulmonary arterial hypertension: a randomized controlled clinical trial. J Am Coll Cardiol 2010; 55: 1915-1922.

10 Simonneau G, Torbicki A, Hoeper MM, et al. Selexipag: an oral, selective prostacyclin receptor agonist for the treatment of pulmonary arterial hypertension. Eur Respir J 2012; 40: 874-880.

11 Sitbon O, Channick R, Chin KM, et al. Selexipag for the treatment of pulmonary arterial hypertension. $N$ Engl $J$ Med 2015; 373: 2522-2533.

12 Galiè N, Brundage BH, Ghofrani HA, et al. Tadalafil therapy for pulmonary arterial hypertension. Circulation 2009; 119: 2894-2903.

13 Galiè N, Ghofrani HA, Torbicki A, et al. Sildenafil citrate therapy for pulmonary arterial hypertension. $N$ Engl $J$ Med 2005; 353: 2148-2157.

14 Rubin LJ, Badesch DB, Barst RJ, et al. Bosentan therapy for pulmonary arterial hypertension. N Engl J Med 2002; 346: 896-903.

15 Gatfield J, Mueller Grandjean C, Sasse T, et al. Slow receptor dissociation kinetics differentiate macitentan from other endothelin receptor antagonists in pulmonary arterial smooth muscle cells. PLoS One 2012; 7: e47662.

16 Pulido T, Adzerikho I, Channick RN, et al. Macitentan and morbidity and mortality in pulmonary arterial hypertension. N Engl J Med 2013; 369: 809-818.

17 Ghofrani HA, Galiè N, Grimminger F, et al. Riociguat for the treatment of pulmonary arterial hypertension. N Engl J Med 2013; 369: 330-340.

18 Ghofrani HA, D'Armini AM, Grimminger F, et al. Riociguat for the treatment of chronic thromboembolic pulmonary hypertension. N Engl J Med 2013; 369: 319-329.

19 Benza RL, Miller DP, Barst RJ, et al. An evaluation of long-term survival from time of diagnosis in pulmonary arterial hypertension from the REVEAL Registry. Chest 2012; 142: 448-456.

20 Stenmark KR, Frid MG, Yeager M, et al. Targeting the adventitial microenvironment in pulmonary hypertension: a potential approach to therapy that considers epigenetic change. Pulm Circ 2012; 2: 3-14.

21 Feng S, Jacobsen SE, Reik W. Epigenetic reprogramming in plant and animal development. Science 2010; 330: 622-627.

22 Kim GH, Ryan JJ, Marsboom G, et al. Epigenetic mechanisms of pulmonary hypertension. Pulm Circ 2011; 1: 347-356.

23 Huang JB, Liang J, Zhao XF, et al. Epigenetics: novel mechanism of pulmonary hypertension. Lung 2013; 191 : 601-610. 
Jin B, Li Y, Robertson KD. DNA methylation: superior or subordinate in the epigenetic hierarchy? Genes Cancer 2011; 2: 607-617.

Paulsen M, Ferguson-Smith AC. DNA methylation in genomic imprinting, development, and disease. J Pathol 2001; 195: 97-110.

Robertson KD. DNA methylation and human disease. Nat Rev Genet 2005; 6: 597-610.

Archer SL, Marsboom G, Kim GH, et al. Epigenetic attenuation of mitochondrial superoxide dismutase 2 in pulmonary arterial hypertension: a basis for excessive cell proliferation and a new therapeutic target. Circulation 2010; 121: 2661-2671.

Yang Q, Lu Z, Ramchandran R, et al. Pulmonary artery smooth muscle cell proliferation and migration in feta lambs acclimatized to high-altitude long-term hypoxia: role of histone acetylation. Am J Physiol Lung Cell Mol Physiol 2012; 303: L1001-L1010.

Perros F, Cohen-Kaminsky S, Gambaryan N, et al. Cytotoxic cells and granulysin in pulmonary arterial hypertension and pulmonary veno-occlusive disease. Am J Respir Crit Care Med 2013; 187: 189-196.

Swygert SG, Peterson CL. Chromatin dynamics: interplay between remodeling enzymes and histone modifications. Biochim Biophys Acta 2014; 1839: 728-736.

Andreoli F, Del Rio A. Physicochemical modifications of histones and their impact on epigenomics. Drug Discov Today 2014; 19: 1372-1379.

Zentner GE, Henikoff S. Regulation of nucleosome dynamics by histone modifications. Nat Struct Mol Biol 2013 20: $259-266$.

Hassan AH, Awad S, Al-Natour Z, et al. Selective recognition of acetylated histones by bromodomains in transcriptional co-activators. Biochem J 2007; 402: 125-133.

Kouzarides T. Acetylation: a regulatory modification to rival phosphorylation? EMBO J 2000; 19: 1176-1179.

$\mathrm{Xu} \mathrm{XF}$, Ma XL, Shen Z, et al. Epigenetic regulation of the endothelial nitric oxide synthase gene in persistent pulmonary hypertension of the newborn rat. J Hypertens 2010; 28: 2227-2235.

Li M, Riddle SR, Frid MG, et al. Emergence of fibroblasts with a proinflammatory epigenetically altered phenotype in severe hypoxic pulmonary hypertension. J Immunol 2011; 187: 2711-2722.

Paulin R, Dromparis P, Sutendra G, et al. Sirtuin 3 deficiency is associated with inhibited mitochondrial function and pulmonary arterial hypertension in rodents and humans. Cell Metab 2014; 20: 827-839.

Meloche J, Pflieger A, Vaillancourt M, et al. Role for DNA damage signaling in pulmonary arterial hypertension. Circulation 2014; 129: 786-797.

Zhao L, Chen CN, Hajji N, et al. Histone deacetylation inhibition in pulmonary hypertension: therapeutic potential of valproic acid and suberoylanilide hydroxamic acid. Circulation 2012; 126: 455-467.

Cantoni S, Galletti M, Zambelli F, et al. Sodium butyrate inhibits platelet-derived growth factor-induced proliferation and migration in pulmonary artery smooth muscle cells through Akt inhibition. FEBS J 2013; 280: 2042-2055.

Bogaard HJ, Mizuno S, Hussaini AA, et al. Suppression of histone deacetylases worsens right ventricular dysfunction after pulmonary artery banding in rats. Am J Respir Crit Care Med 2011; 183: 1402-1410.

Terbach N, Williams RS. Structure-function studies for the panacea, valproic acid. Biochem Soc Trans 2009; 37: $1126-1132$.

Cavasin MA, Demos-Davies K, Horn TR, et al. Selective class I histone deacetylase inhibition suppresses hypoxia-induced cardiopulmonary remodeling through an antiproliferative mechanism. Circ Res 2012; 110: 739-748.

Csiszar A, Labinskyy N, Olson S, et al. Resveratrol prevents monocrotaline-induced pulmonary hypertension in rats. Hypertension 2009; 54: 668-675.

Paffett ML, Lucas SN, Campen MJ. Resveratrol reverses monocrotaline-induced pulmonary vascular and cardiac dysfunction: a potential role for atrogin-1 in smooth muscle. Vascul Pharmacol 2012; 56: 64-73.

Aljubran SA, Cox R Jr, Tamarapu Parthasarathy P, et al. Enhancer of zeste homolog 2 induces pulmonary artery smooth muscle cell proliferation. PLoS One 2012; 7: e37712.

Yang Q, Lu Z, Singh D, et al. BIX-01294 treatment blocks cell proliferation, migration and contractility in ovine foetal pulmonary arterial smooth muscle cells. Cell Prolif 2012; 45: 335-344.

Nagano T, Fraser P. No-nonsense functions for long noncoding RNAs. Cell 2011; 145: 178-181.

Carthew RW, Sontheimer EJ. Origins and mechanisms of miRNAs and siRNAs. Cell 2009; 136: 642-655.

Bartel DP. MicroRNAs: genomics, biogenesis, mechanism, and function. Cell 2004; 116: 281-297.

Ambros V, Bartel B, Bartel DP, et al. A uniform system for microRNA annotation. RNA 2003; 9: 277-279.

Kozomara A, Griffiths-Jones S. miRBase: integrating microRNA annotation and deep-sequencing data. Nucleic Acids Res 2011; 39: D152-D157.

Mathelier A, Carbone A. Large scale chromosomal mapping of human microRNA structural clusters. Nucleic Acids Res 2013; 41: 4392-4408.

Lu M, Zhang Q, Deng M, et al. An analysis of human microRNA and disease associations. PLoS One 2008; 3: e3420. Caruso P, MacLean MR, Khanin R, et al. Dynamic changes in lung microRNA profiles during the development of pulmonary hypertension due to chronic hypoxia and monocrotaline. Arterioscler Thromb Vasc Biol 2010; 30: 716-723.

Schlosser K, Taha M, Deng Y, et al. Discordant regulation of microRNA between multiple experimental models and human pulmonary hypertension. Chest 2015; 148: 481-490.

Bertero T, Lu Y, Annis S, et al. Systems-level regulation of microRNA networks by miR-130/301 promotes pulmonary hypertension. J Clin Invest 2014; 124: 3514-3528.

Chen Z, Nakajima T, Tanabe N, et al. Susceptibility to chronic thromboembolic pulmonary hypertension may be conferred by miR-759 via its targeted interaction with polymorphic fibrinogen alpha gene. Hum Genet 2010; 128: $443-452$.

Guo L, Yang Y, Liu J, et al. Differentially expressed plasma microRNAs and the potential regulatory function of Let-7b in chronic thromboembolic pulmonary hypertension. PLoS One 2014; 9: e101055.

Rhodes CJ, Wharton J, Boon RA, et al. Reduced microRNA-150 is associated with poor survival in pulmonary arterial hypertension. Am J Respir Crit Care Med 2013; 187: 294-302.

Pullamsetti SS, Doebele C, Fischer A, et al. Inhibition of microRNA-17 improves lung and heart function in experimental pulmonary hypertension. Am J Respir Crit Care Med 2012; 185: 409-419. 

hypoxia. Biochem J 2014; 462: 103-112.

63 Yang S, Banerjee S, Freitas A, et al. miR-21 regulates chronic hypoxia-induced pulmonary vascular remodeling. Am J Physiol Lung Cell Mol Physiol 2012; 302: L521-L529.

64 Kang BY, Park KK, Green DE, et al. Hypoxia mediates mutual repression between microRNA-27a and PPAR $\gamma$ in the pulmonary vasculature. PLoS One 2013; 8: e79503.

65 Doebele C, Bonauer A, Fischer A, et al. Members of the microRNA-17-92 cluster exhibit a cell-intrinsic antiangiogenic function in endothelial cells. Blood 2010; 115: 4944-4950.

66 Kim J, Kang Y, Kojima Y, et al. An endothelial apelin-FGF link mediated by miR-424 and miR-503 is disrupted in pulmonary arterial hypertension. Nat Med 2013; 19: 74-82.

67 Bertero T, Cottrill K, Krauszman A, et al. The microRNA-130/301 family controls vasoconstriction in pulmonary hypertension. J Biol Chem 2015; 290: 2069-2085.

68 Courboulin A, Paulin R, Giguère NJ, et al. Role for miR-204 in human pulmonary arterial hypertension. $J$ Exp Med 2011; 208: 535-548.

69 Meloche J, Potus F, Vaillancourt M, et al. Bromodomain-containing protein 4: the epigenetic origin of pulmonary arterial hypertension. Circ Res 2015; 117: 525-535.

70 Yue J, Guan J, Wang X, et al. MicroRNA-206 is involved in hypoxia-induced pulmonary hypertension through targeting of the HIF-1 $\alpha /$ Fhl-1 pathway. Lab Invest 2013; 93: 748-759.

71 Jalali S, Ramanathan GK, Parthasarathy PT, et al. Mir-206 regulates pulmonary artery smooth muscle cell proliferation and differentiation. PLoS One 2012; 7: e46808.

72 Shan F, Li J, Huang QY. HIF-1 alpha-induced up-regulation of miR-9 contributes to phenotypic modulation in pulmonary artery smooth muscle cells during hypoxia. J Cell Physiol 2014; 229: 1511-1520.

73 Li S, Ran Y, Zhang D, et al. MicroRNA-138 plays a role in hypoxic pulmonary vascular remodelling by targeting Mst1. Biochem J 2013; 452: 281-291.

74 Guo L, Qiu Z, Wei L, et al. The microRNA-328 regulates hypoxic pulmonary hypertension by targeting at insulin growth factor 1 receptor and L-type calcium channel- $\alpha 1$ C. Hypertension 2012; 59: 1006-1013.

75 Meloche J, Le Guen M, Potus F, et al. miR-223 reverses experimental pulmonary arterial hypertension. Am J Physiol Cell Physiol 2015; 309: C363-C372.

76 Zeng Y, Zhang X, Kang K, et al. MicroRNA-223 attenuates hypoxia-induced vascular remodeling by targeting RhoB/MLC2 in pulmonary arterial smooth muscle cells. Sci Rep 2016; 6: 24900.

77 Shi L, Kojonazarov B, Elgheznawy A, et al. miR-223-IGF-IR signalling in hypoxia- and load-induced right-ventricular failure: a novel therapeutic approach. Cardiovasc Res 2016; 111: 184-193

78 Wang L, Guo LJ, Liu J, et al. MicroRNA expression profile of pulmonary artery smooth muscle cells and the effect of let-7d in chronic thromboembolic pulmonary hypertension. Pulm Circ 2013; 3: 654-664.

79 Wang $\mathrm{D}$, Zhang $\mathrm{H}$, Li M, et al. MicroRNA-124 controls the proliferative, migratory, and inflammatory phenotype of pulmonary vascular fibroblasts. Circ Res 2014; 114: 67-78.

80 Kang K, Peng X, Zhang X, et al. MicroRNA-124 suppresses the transactivation of nuclear factor of activated T cells by targeting multiple genes and inhibits the proliferation of pulmonary artery smooth muscle cells. $J$ Biol Chem 2013; 288: 25414-25427.

81 Potus F, Ruffenach G, Dahou A, et al. Downregulation of microRNA-126 contributes to the failing right ventricle in pulmonary arterial hypertension. Circulation 2015; 132: 932-943.

82 Paulin R, Sutendra G, Gurtu V, et al. A miR-208-Mef2 axis drives the decompensation of right ventricular function in pulmonary hypertension. Circ Res 2015; 116: 56-69.

83 Kuehbacher A, Urbich C, Zeiher AM, et al. Role of Dicer and Drosha for endothelial microRNA expression and angiogenesis. Circ Res 2007; 101: 59-68.

84 White K, Dempsie Y, Caruso P, et al. Endothelial apoptosis in pulmonary hypertension is controlled by a microRNA/programmed cell death 4/caspase-3 axis. Hypertension 2014; 64: 185-194.

85 Brock M, Trenkmann M, Gay RE, et al. Interleukin-6 modulates the expression of the bone morphogenic protein receptor type II through a novel STAT3-microRNA cluster 17/92 pathway. Circ Res 2009; 104: 1184-1191.

86 Bonauer A, Carmona G, Iwasaki M, et al. MicroRNA-92a controls angiogenesis and functional recovery of ischemic tissues in mice. Science 2009; 324: 1710-1713.

87 Albinsson S, Suarez Y, Skoura A, et al. MicroRNAs are necessary for vascular smooth muscle growth, differentiation, and function. Arterioscler Thromb Vasc Biol 2010; 30: 1118-1126.

88 Sarkar J, Gou D, Turaka P, et al. MicroRNA-21 plays a role in hypoxia-mediated pulmonary artery smooth muscle cell proliferation and migration. Am J Physiol Lung Cell Mol Physiol 2010; 299: L861-L871.

89 Kwapiszewska G, Wygrecka M, Marsh LM, et al. Fhl-1, a new key protein in pulmonary hypertension. Circulation 2008; 118: 1183-1194.

90 Ponting CP, Oliver PL, Reik W. Evolution and functions of long noncoding RNAs. Cell 2009; 136: 629-641.

91 Wapinski O, Chang HY. Long noncoding RNAs and human disease. Trends Cell Biol 2011; 21 : 354-361.

92 Ziats MN, Rennert OM. Aberrant expression of long noncoding RNAs in autistic brain. J Mol Neurosci 2013; 49: 589-593.

93 Kogo R, Shimamura T, Mimori K, et al. Long noncoding RNA HOTAIR regulates polycomb-dependent chromatin modification and is associated with poor prognosis in colorectal cancers. Cancer Res 2011; 71: $6320-6326$.

94 Ling H, Vincent K, Pichler M, et al. Junk DNA and the long non-coding RNA twist in cancer genetics. Oncogene 2015; 34: 5003-5011.

95 Greco S, Gorospe M, Martelli F. Noncoding RNA in age-related cardiovascular diseases. J Mol Cell Cardiol 2015; 83: $142-155$.

96 Vencken SF, Greene CM, McKiernan PJ. Non-coding RNA as lung disease biomarkers. Thorax 2015; 70: 501-503.

97 Wang X, Yan C, Xu X, et al. Long noncoding RNA expression profiles of hypoxic pulmonary hypertension rat model. Gene 2016; 579: 23-28.

$98 \mathrm{Gu}$ S, Li G, Zhang X, et al. Aberrant expression of long noncoding RNAs in chronic thromboembolic pulmonary hypertension. Mol Med Rep 2015; 11: 2631-2643. 
Josipovic I, Fork C, Preussner J, et al. PAFAH1B1 and the lncRNA NONHSAT073641 maintain an angiogenic phenotype in human endothelial cells. Acta Physiol 2016 (In press DOI: 10.1111/alpha.12700)

$100 \mathrm{Xu} \mathrm{XF}, \mathrm{Lv} \mathrm{Y}, \mathrm{Gu}$ WZ, et al. Epigenetics of hypoxic pulmonary arterial hypertension following intrauterine growth retardation rat: epigenetics in PAH following IUGR. Respir Res 2013; 14: 20.

101 Chen D, Fang F, Yang Y, et al. Brahma-related gene 1 (Brg1) epigenetically regulates CAM activation during hypoxic pulmonary hypertension. Cardiovasc Res 2013; 100: 363-373.

102 Chen D, Yang Y, Cheng X, et al. Megakaryocytic leukemia 1 directs a histone H3 lysine 4 methyltransferase complex to regulate hypoxic pulmonary hypertension. Hypertension 2015; 65: 821-833.

103 Nan X, Ng HH, Johnson CA, et al. Transcriptional repression by the methyl-CpG-binding protein MeCP2 involves a histone deacetylase complex. Nature 1998; 393: 386-389.

104 Hendrich B, Tweedie S. The methyl-CpG binding domain and the evolving role of DNA methylation in animals. Trends Genet 2003; 19: 269-277.

105 Rottach A, Leonhardt H, Spada F. DNA methylation-mediated epigenetic control. J Cell Biochem 2009; 108: 43-51.

106 Ooi SK, Qiu C, Bernstein E, et al. DNMT3L connects unmethylated lysine 4 of histone H3 to de novo methylation of DNA. Nature 2007; 448: 714-717.

107 Otani J, Nankumo T, Arita K, et al. Structural basis for recognition of H3K4 methylation status by the DNA methyltransferase 3A ATRX-DNMT3-DNMT3L domain. EMBO Rep 2009; 10: 1235-1241.

108 Wu L, Zhou H, Zhang Q, et al. DNA methylation mediated by a microRNA pathway. Mol Cell 2010; 38: 465-475.

109 Kelly TK, De Carvalho DD, Jones PA. Epigenetic modifications as therapeutic targets. Nat Biotechnol 2010; 28: 1069-1078.

110 Janssen HL, Reesink HW, Lawitz EJ, et al. Treatment of HCV infection by targeting microRNA. N Engl J Med 2013; 368: 1685-1694.

111 Kim J, Hwangbo C, Hu X, et al. Restoration of impaired endothelial myocyte enhancer factor 2 function rescues pulmonary arterial hypertension. Circulation 2015; 131: 190-199.

112 Lall S. Primers on chromatin. Nat Struct Mol Biol 2007; 14: 1110-1115. 